UCRL-JC-115937

PREPRINT

\title{
Detailed Measurements and Shaping of Gate Profiles for Microchannel-Plate-Based X-ray Framing Cameras
}

\author{
O. L. Landen, A. Abare, B. A. Hammel, P. M. Bell, D. K. Bradley \\ RECEIVED \\ NOV - 31995 \\ OSTI
}

This paper was prepared for submittal to the

SPIE International Symposium on Optical

Instrumentation and Applied Science

San Diego, CA

July 24-29, 1994

October 3, 1994
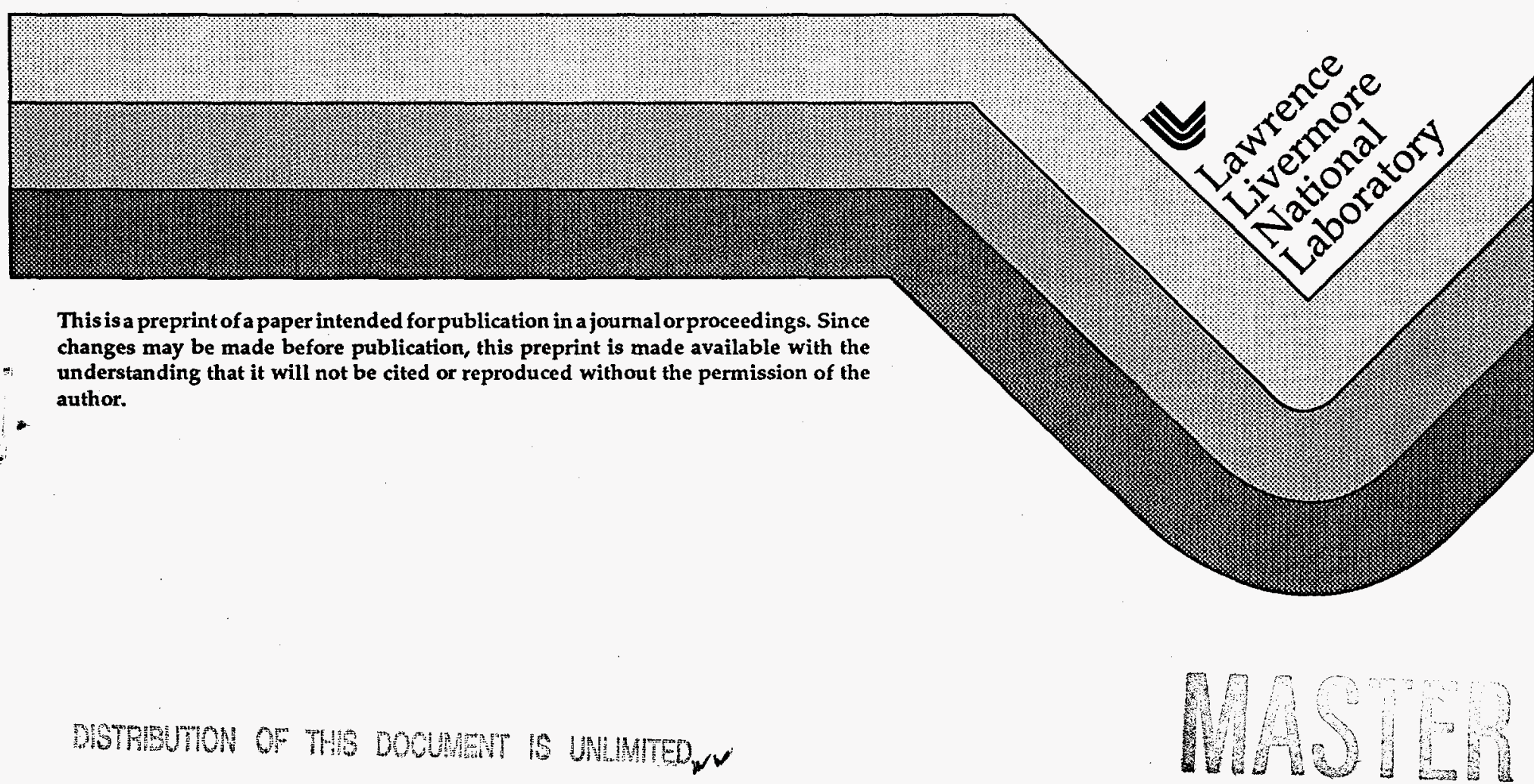


\section{DISCLAIMER}

This document was prepared as an account of work sponsored by an agency of the United States Government. Neither the United States Government nor the University of California nor any of their employees, makes any warranty, express or implied, or assumes any legal liability or responsibility for the accuracy, completeness, or usefulness of any information, apparatus, product, or process disclosed, or represents that its use would not infringe privately owned rights. Reference herein to any specific commercial product, process, or service by trade name, trademark, manufacturer, or otherwise, does not necessarily constitute or imply its endorsement, recommendation, or favoring by the United States Government or the University of California. The views and opinions of authors expressed herein do not necessarily state or reflect those of the United States Government or the University of California, and shall not be used for advertising or product endorsement purposes. 


\section{DISCLAIMER}

Portions of this document may be illegible electronic image products. Images are produced from the best available original document. 
Detailed measurements and shaping of gate profiles for microchannel plate-based x-ray framing cameras

\author{
O.L. Landen, A. Abare\#, B.A. Hammel, P.M. Bell, and D.K. Bradley* \\ Lawrence Livermore National Laboratory, P.O. Box 5508, Livermore, CA 94550 \\ \#University of Florida, Gainesville, FL 32607 \\ * Laboratory for Laser Energetics, University of Rochester, 250 E. River Road, Rochester, NY 14623
}

\begin{abstract}
Large dynamic range (> 1000) measurements of gain profiles for gated microchannel plates (MCP) are presented. Temporal profiles are reconstructed for any point on the microstrip transmission line from data acquired over many shots with variable delay. No evidence for significant pulse distortion by voltage reflections at the ends of the microstrip is observed. The measured profiles compare well to predictions by a time-dependent discrete dynode model down to the $1 \%$ level. The calculations do overestimate the contrast further into the temporal wings. The role of electron transit time dispersion in limiting the minimum achievable gate duration is then investigated by using variable duration flattop gating pulses. A minimum gate duration of $50 \mathrm{ps}$ is achieved with flattop gating, consistent with a fractional transit time spread of $\approx 15 \%$.
\end{abstract}

Keywords: microchannel-plate, $x$-ray framing camera, microstrip

\title{
1. INTRODUCTION
}

Gated, microchannel-plate-based (MCP) framing cameras ${ }^{1-4}$ are increasingly used worldwide for $x$-ray imaging of subnanosecond laser-plasma phenomena. The camera shutter is provided by launching a short ( $\approx 200 \mathrm{ps)} 1 \mathrm{kV}$ voltage pulse across a microstrip transmission line coated on the microchannel plate. A photoelectron signal produced at the front surface of the MCP is then only amplified during the transit time of the voltage pulse across a given point on the microstrip: In some cases, the $x$-ray source duration may not be much longer than the minimum gate times currently available (40 - 100 ps full width at half maximum (FWHM)). A correct interpretation of such transient images (e.g. $x$-ray core images from inertial confinement fusion (ICF) implosions) then requires accurate knowledge of the camera temporal gate profile, especially in the wings. Accordingly, detailed, high dynamic range $(>1000)$ unsaturated measurements of gain profiles for a typical 90 ps FWHM resolution camera have been performed over a nanosecond range using high contrast ps UV illumination.

In addition, the constant quest for higher temporal resolution (and, indirectly, higher spatial resolution if motional blurring is an issue) in x-ray imaging of high temperature plasma phenomena has led to the deployment of increasingly shorter gate widths $1,3,5$ for microchannel-plate-based X-ray framing cameras. Robust reductions in gate FWHM from 90 ps to 35 ps have been achieved by simply using thinner plates and shorter high-voltage gating pulses with no special emphasis on voltage pulse profile optimization. Surprisingly, even for the shorter gate profiles, measurements show good agreement with a simple discrete dynode model $^{6}$ which ignores electron transit time dispersion and is only strictly valid for slowly varying gate voltages $^{5}$. In an attempt to either validate the predictive capabilities of this simple model for future higher-speed or higher gate contrast camera development or to explain a systematic fortuitous agreement possibly arising from the self-similar nature of the gate profiles used up to now, gate profile measurements were performed using quasi-flat-top gate voltage profiles with continuously adjustable amplitude and FWHM. Measured gain profiles were then compared to a discrete dynode model ignoring transit time dispersion.

\section{HIGH CONTRAST GATE PROFILE MEASUREMENTS}

\subsection{Experimental technique}

Previous measurements of gate profile FWHM have often used a single-shot technique ${ }^{2,7-9}$ which freezes the gain profile vs. position by illuminating the MCP with a diffuse short pulse UV laser beam. The spatial extent of the image is then translated into time by using auxiliary measurements of the pulse propagation velocity. This method suffers from three disadvantages: the 
gate profile derived is only approximate because the peak gain varies with position due to resistive propagation losses ${ }^{5,7}$, the gain profile FWHM can only be monitored when the image is near the center of the microstrip because of the finite extent of a typical microstrip ( 200 ps or $\approx$ two FWHM), and accurate measurements of the gate propagation velocity are difficult. Moreover, even when the image is centered, only measurements extending $\pm 100 \mathrm{ps}$ on either side of the peak are possible.

Hence, for performing the high contrast gate profile measurements at each point on the microstrip which are relevant to correctly interpreting ICF images, a multiple strobe shot technique ${ }^{8}$ must be used. Moreover, the jitter between the exciting laser pulse and the camera trigger is large (up to $\pm 50 \mathrm{ps}$ ). Hence, when the position of peak gain is timed to be off the microstrip, a low jitter temporal fiducial is also necessary. A schematic of the experimental technique providing low jitter strobe measurements is shown in Fig. 1. A common trigger was used to launch the desired gating voltage pulse (denoted GXI2 and shown in Fig. 2) and a reference pulse along separate microstrips coated on the same MCP (LD $=45$ with $D=10 \mu \mathrm{m}$ pores). The residual jitter between pulsers was measured at $\pm 20 \mathrm{ps}$, adequate for present purposes. Known, variable delays between the pulsers was achieved by using a precalibrated $50 \Omega$ electrical trombone inserted in the GXI2 pulser path. By positioning the peak gain of the reference image at the strip center, a temporal fiducial was always visible. The two microstrips were uniformly illuminated ( $\pm 20 \%$ variations) with spatially-smoothed, $203 \mathrm{~nm}, 3$ ps laser pulses 7,10 . The MCP images were recorded by a fiber-optically-coupled CCD camera and stored on computer for further analysis. On each shot the laser energy was monitored for shot-to-shot signal normalization.

\section{3 ps UV illumination}

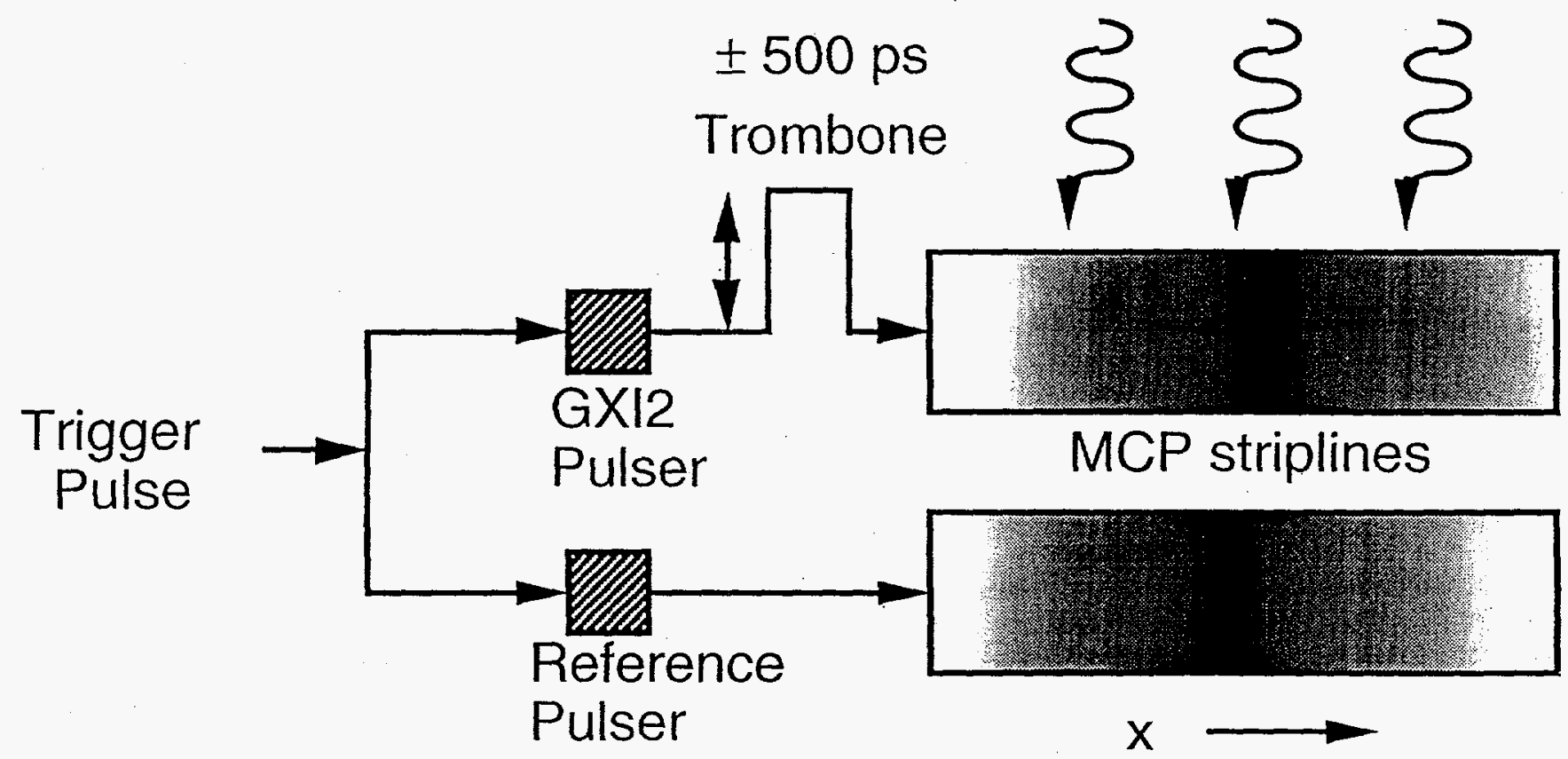

Figure 1 Experimental set-up for measuring gate profiles

Several auxiliary measurements were performed to assure instrumental effects were not leading to inaccurate records of the gate profiles. The laser light intensity distribution across the MCP, measured by operating the camera in dc mode, was shown to be essentially constant from shot-to-shot ( $\pm 10 \%$ variations). The laser intensity was kept low enough to avoid MCP saturation and varied to demonstrate linearity ${ }^{7}$. The laser pulses traversing the same optics were monitored by a well characterized $x$-ray streak camera ${ }^{10}$ operated at $10 \mathrm{ps}$ resolution and at a dynamic range of 3000 to check for the level of pre-and post-pulses which would limit the measurable gate contrast. A streak record of a typical laser pulse (Fig. 3a) shows a slight post-pulse extending up to +50 ps. The convolution of such a sampling pulse with a simulated 80 ps Gaussian FWHM gate profile is shown on a semi-log plot in Figure 3b. A comparison of Fig. 3b with the actual measured profiles (e.g. Fig. 4) shows that the laser pulse profile did not affect the measured gate profiles. 


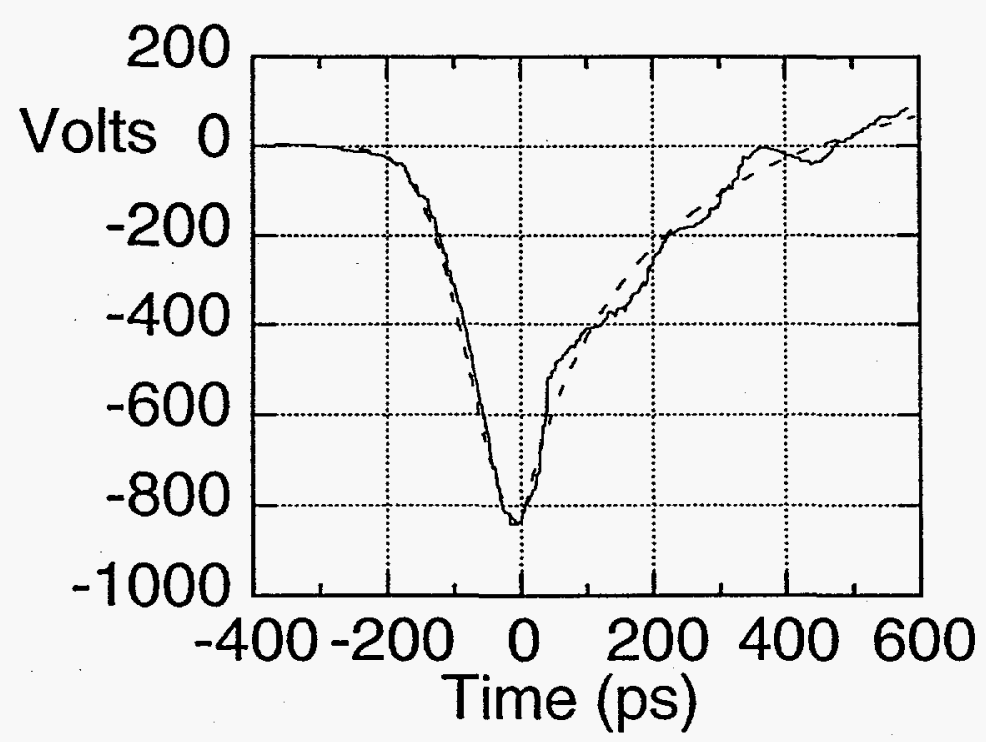

Figure 2 Measured gate voltage profile (solid curve) and analytic fit used in gain modelling (dashed curve).
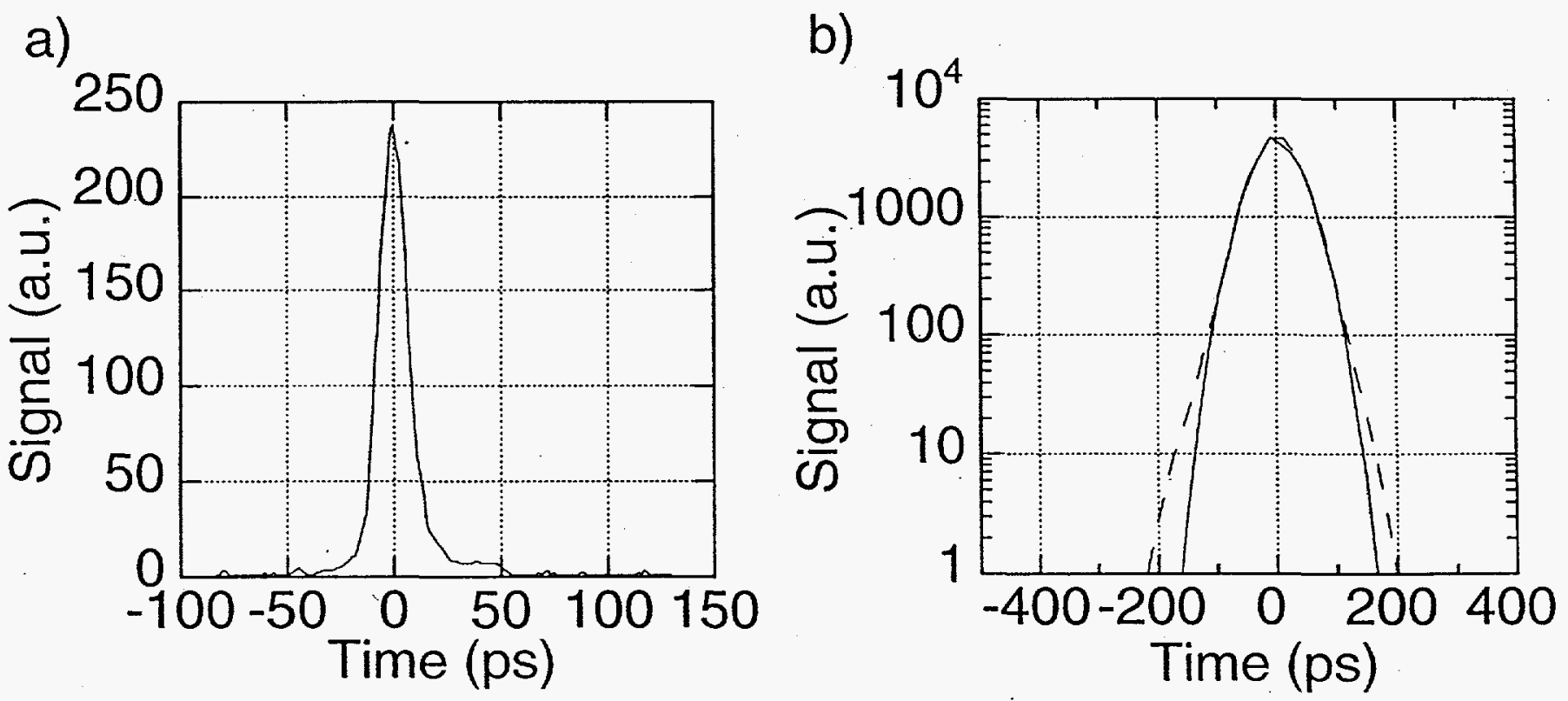

Figure 3 a) 10 ps resolution streak record of 3 ps UV laser pulse showing minor post-pulse. b) Simulated 80ps Gaussian FWHM gate profile (solid curve) convolved with laser pulse profile shown in a) (dashed curve).

For each shot, the relative gain $G(x, \Delta t)$ was calculated by dividing the measured signal distribution $I(x, \Delta t)$ by the laser intensity distribution $L(x)$ and the laser energy. For each position $x$, the gain profile $G(\Delta t)$ was then reconstructed from many shots fired at different delays $\Delta t$. 


\subsection{Results}

Typical high contrast gain profiles for the gating voltage pulse shown in Fig. 2 are shown on a semi-log plot in Fig. 4. The two sets of data differ by the level of forward dc bias added to the pulsed voltage $(-100$ and $-300 \mathrm{~V})$. These levels of bias are typical in ICF usage. The gain axis is relative but consistent for the two sets of data. The leading edge of the profile is at negative delay and the profiles shown here were taken near the center of the microstrip. The shutter contrasts reach 1000 at $300-$ 400 ps delay relative to the peak. Note that the measured FWHM, though less accurate and not the focus of this study, are $\approx$ $80-100$ ps, consistent with earlier single-shot low contrast measurements?

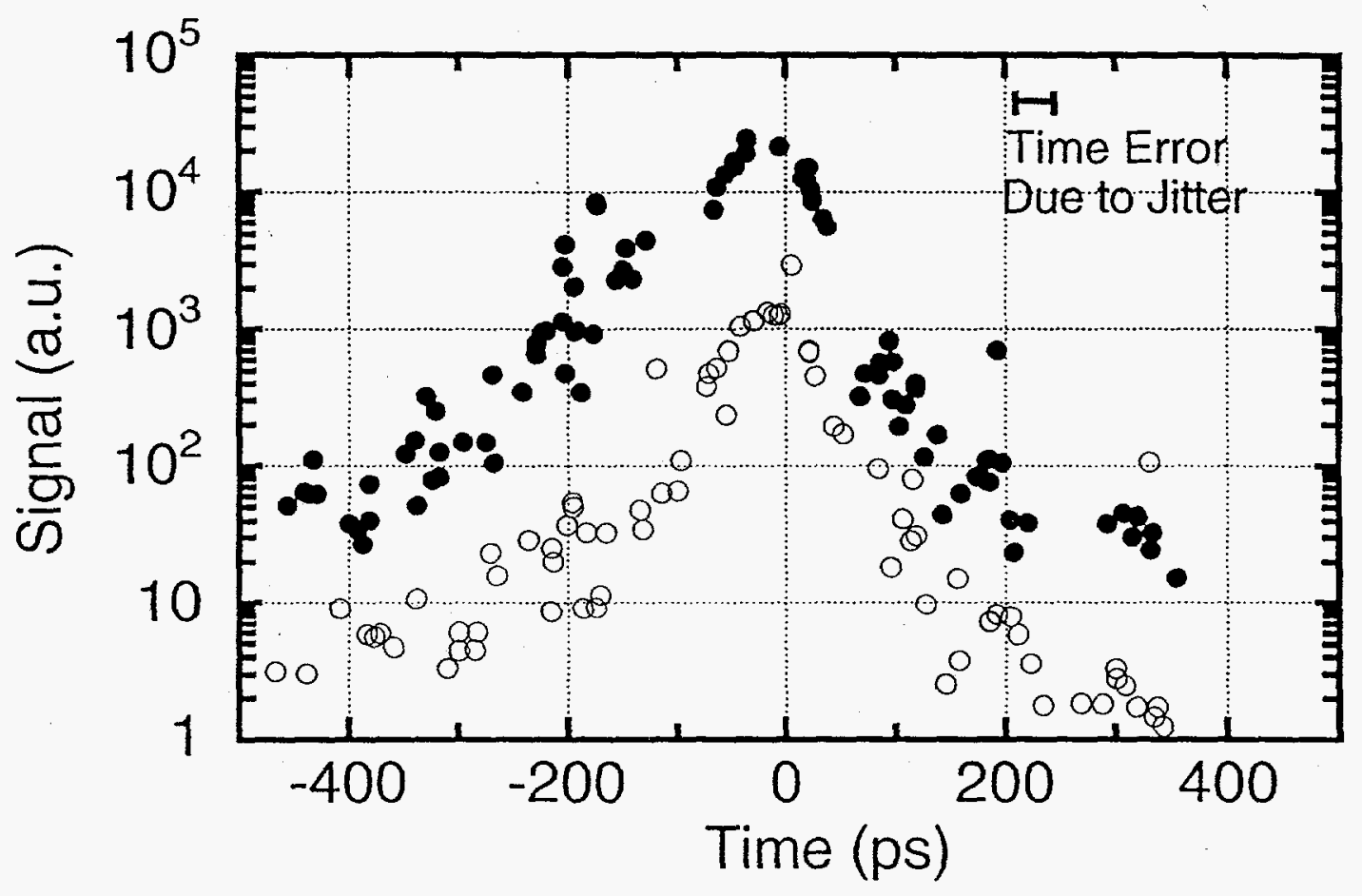

Figure 4. Measured gate profiles near center of stripline $(x=13.4 \mathrm{~mm})$ for gate voltage profile shown in Fig. 2 with additional $-100 \mathrm{~V}$ (open circles) and $-300 \mathrm{~V}$ (closed circles) forward dc bias applied.

Plots of the gain profile for three positions along the microstrip corresponding to the beginning ( $1.4 \mathrm{~mm}$ ), middle (13.4 mm) and end $(25.9 \mathrm{~mm})$ are shown in Fig. 5 for the $300 \mathrm{~V}$ additional dc bias case. The profiles have been displaced by three decades in the ordinate for clarity and show the usual peak gain degradation ${ }^{7}$ with position as the pulse suffers resistive losses during propagation. The data has been fitted by simple analytic curves to aid the eye, normalized to unity at peak gain and replotted in the reference frame of the propagating voltage pulse on Fig. 6 . The profile shapes show little sensitivity to position and suggest that the effects of voltage reflections which would be expected to be most noticeable at the ends of the microstrip are not a major source of profile distortion. There is, however, evidence of loss of contrast at the 300:1 contrast level as the pulse reaches the center of the microstrip for the high bias case (Fig. 6b). This may be due to a small amount of pulse dispersion allowing the total voltage (pulsed $+-300 \mathrm{~V}$ dc bias) to exceed the threshold voltage for unity gain $5(\approx-400 \mathrm{~V})$ further out in the temporal wings. For the $-100 \mathrm{~V}$ bias case, -300 rather than $-100 \mathrm{~V}$ pulsed would be needed to exceed unity gain, a much less likely proposition which might explain the lack of shape variation with position shown in Fig. 6 a. 


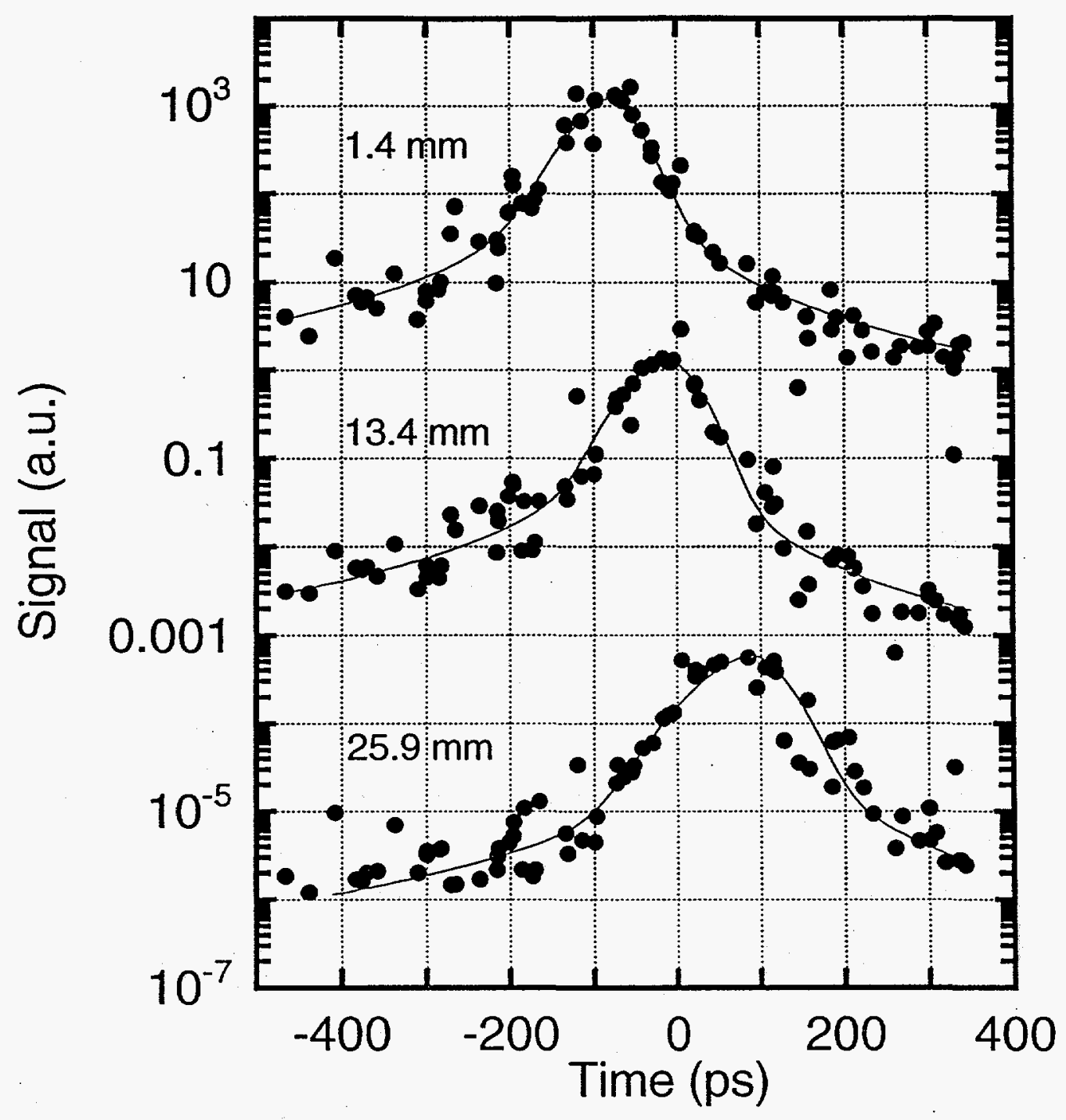

Figure 5 Gain profiles versus position on MCP stripline for pulsed voltage shown in Fig. 2 with additional $-300 \mathrm{~V}$ forward dc bias applied. Curves are fits using combinations of asymmetric Gaussian and Lorentzian functions.

\subsection{Modelling}

The gain profiles were simulated using a time-dependent discrete dynode mode1 ${ }^{5,6}$. The instantaneous gain per amplification stage (dynode) is given by:

$$
\mathrm{G}(\mathrm{t})=\left(\mathrm{V}(\mathrm{t}) / \mathrm{nV}_{\mathrm{c}}\right)^{0.5}
$$

where $n$ is the number of dynodes, $V(t)$ is the instantaneous applied voltage (pulsed $+d c$ ), and $V_{c}$ is the threshold voltage for unity gain. The total gain under dc conditions (constant $V$ ) is hence proportional to $V^{0} .5 n$. Dc measurements of gain vs. voltage ${ }^{7}$ yielded a $\mathrm{V}^{13}$ dependence suggesting $n=26 . V_{c}$ was then estimated at $15 \mathrm{~V}$ based on the measured voltage required for the onset of MCP saturation during single photoelectron detection. The cumulative gain is calculated by applying Eq. (1) 
dynode-by-dynode as a function of the birth time of a photoelectron, assuming no transit time dispersion. The pulsed contribution to $\mathrm{V}(\mathrm{t})$ is taken from a smooth analytic fit to the measured voltage profile, shown as the dashed curve in Fig. 2 and given by:

$$
\begin{array}{ll}
V(t)=-840 \exp \left[-(t / 90)^{2} \ln 2\right] & t<0 \\
V(t)=-1.42[840 /(1+t / 190)-250] & t>0
\end{array}
$$

with $t$ in ps.
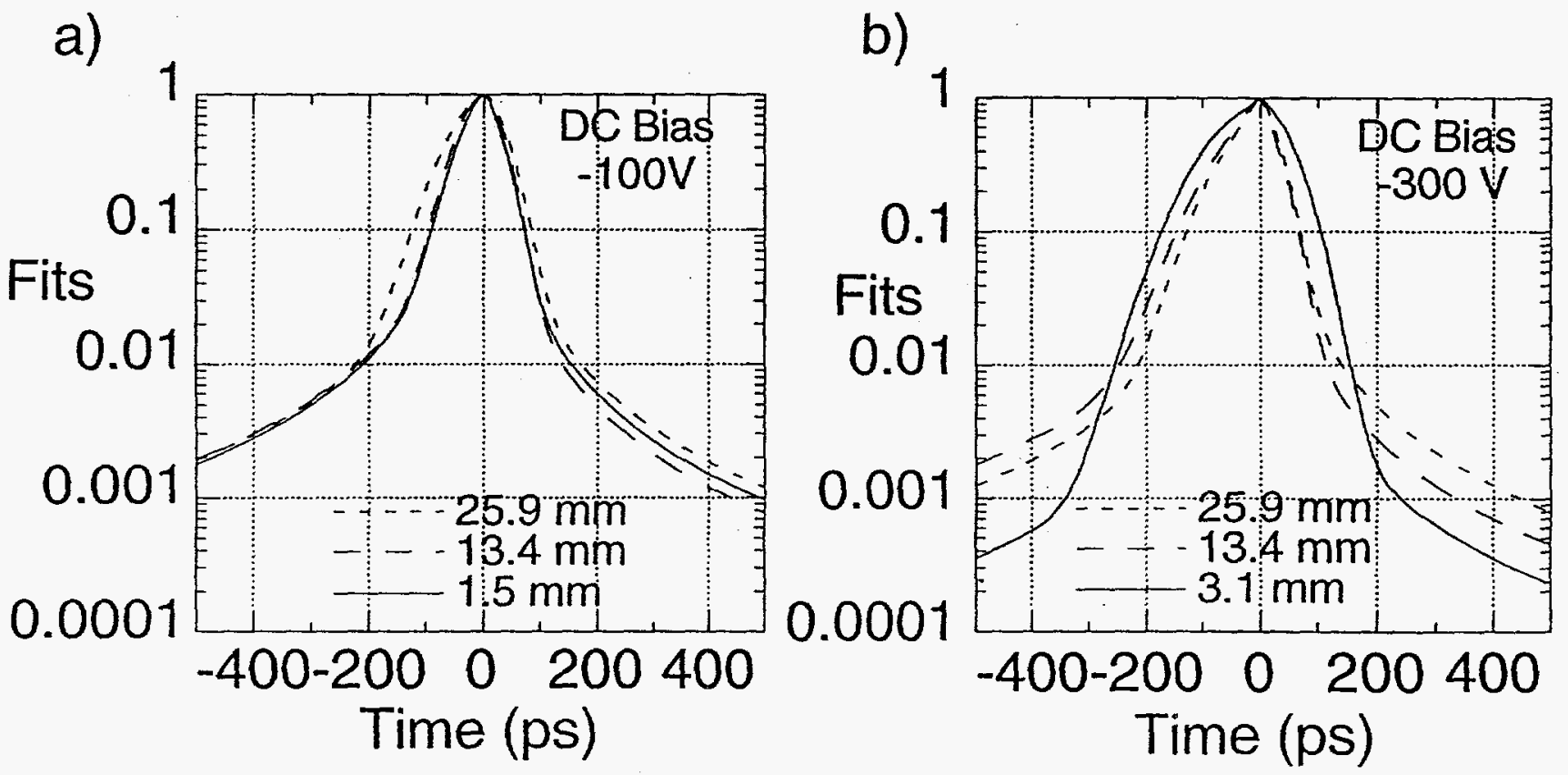

Figure 6 Fits to gate profiles for start $(1.5-3.1 \mathrm{~mm})$, middle $(13.4 \mathrm{~mm})$ and end $(25.9 \mathrm{~mm})$ of stripline. Peak signals are normalized to unity and profiles plotted in reference frame of propagating gate pulse (peak set at $t=0$ ). a) $-100 \mathrm{~V}$ and b)-300 V forward dc bias added to pulsed voltage shown in Fig. 2.

The simulation results, overplotted as curves on profile data acquired at the beginning of the microstrip where voltage dispersion effects should be minimal, are shown on Fig. 7. The simulations have been normalized in both magnitude and timing to the peak gain data. The simulated profiles are insensitive to the exact value of $V_{c}$. Negative delay corresponds to photoelectrons born early. Good agreement between measured and calculated gain profiles is observed for intensity levels as low as $1 \%$ of the peak. Moreover, the observation of a slower risetime than fall-time is accurately predicted. The discrepancies at lower signal levels at late delays are attributed to neglecting secondary electron transit time dispersion 6 . Specifically, the majority of the signal at late delay ( $>200$ ps after peak gain) can be attributed to secondary electrons transiting the MCP more quickly than the average thereby still benefitting from the rapidly dropping gain on the tail of the voltage pulse. A simple statistical estimate $^{6}$ for the average transit time dispersion is $15 \%$ of the 300 ps transit time, $\approx 50$ ps. This is consistent with the e-folding time for the measured gain profiles at large positive delay. It is also small enough that it would be an undetectable source of broadening above the $1 \%$ intensity level when added in quadrature on a semi-log plot with the predicted FWHM of 90 100 ps. 

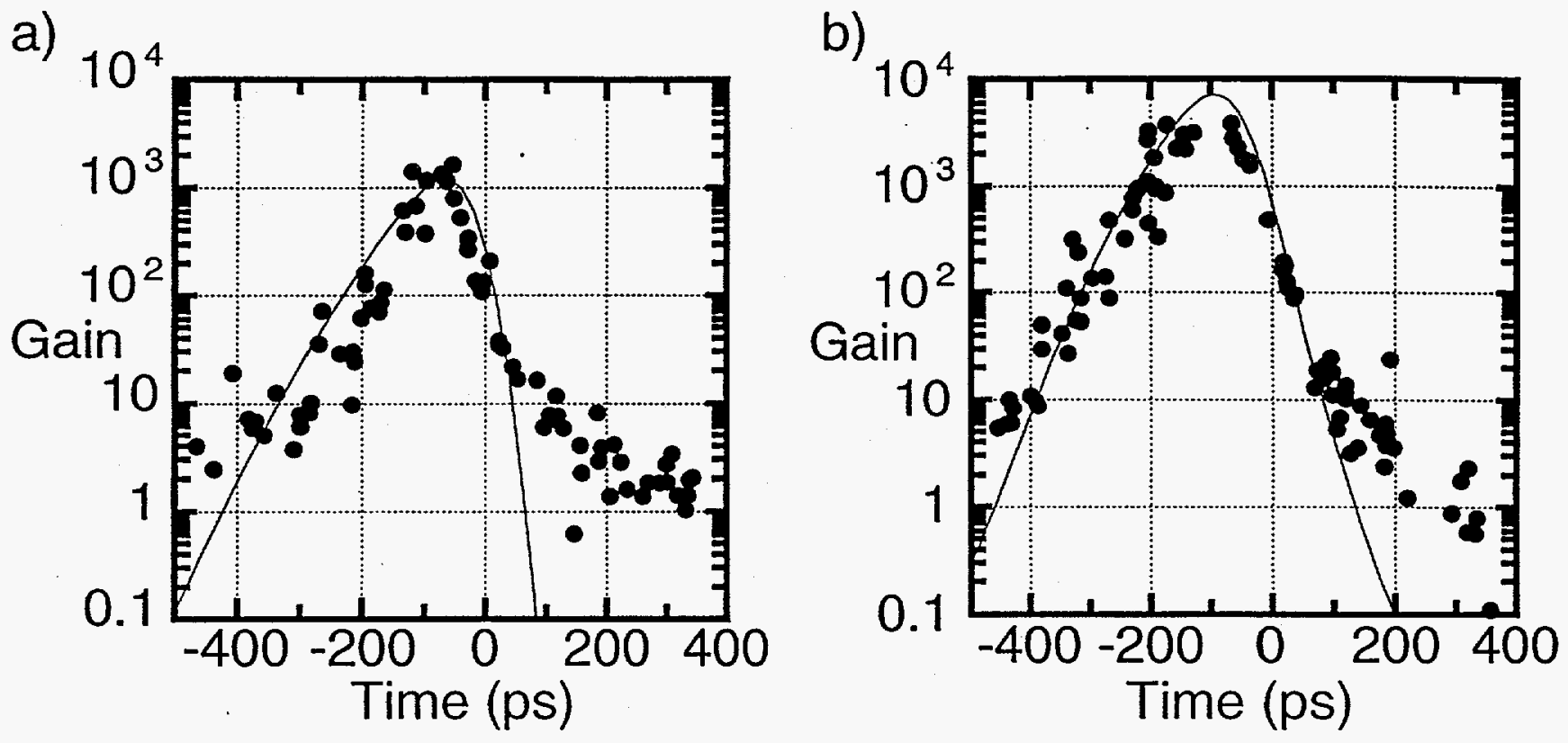

Figure 7 Measured (circles) and calculated gain profiles (curves) at $x=1.4 \mathrm{~mm}$ for pulsed gate voltage shown in Fig. 2 with additional a) $-100 \mathrm{~V}$ and b) $-300 \mathrm{~V}$ forward $\mathrm{DC}$ bias applied.

\section{FLAT-TOP GATE PROFILES}

\subsection{Profile shaping}

A comparison of the data and simulations depicted in Fig. 7 only shows evidence of transit time dispersion far from the profile peak. Clearly, voltage profiles for which gate FWHM shorter than $50 \mathrm{ps}$ are predicted in the absence of dispersion are needed for a better test of the effects of dispersion. Traditional gated MCP cameras have operated on the principle that their Gaussian-like voltage pulses provide gain narrowing ${ }^{5,8}$ of $\sqrt{n}_{\mathrm{n}} / 2 \approx 3.5 \mathrm{x}$. However such pulses can only provide useful gain levels when the voltage FWHM is greater than the 300 ps transit time, hence limiting the minimum useful gate FWHM to $\approx$ 80 ps. For shorter gate widths, a top-hat gating pulse with a FWHM slightly greater than the secondary electron transit time might be the ideal choice. In this case, the feature of gain narrowing is dropped in favor of maintaining high gain during the full amplification time of those photoelectrons born at the leading edge of the gating pulse. Photoelectrons (and the secondary electrons they produce) that are born slightly earlier (later) will experience no gain at the first (last) dynodes, hence in principle providing a rapid shutter.

Flat-top voltage profiles of $-1.25 \mathrm{kV}$ maximum amplitude and variable FWHM were passively engineered by combining an electronically synchronized but delayed and inverted $3.8 \mathrm{kV}, 90 \mathrm{ps}$ risetime step pulse with its twin output. A schematic of the shaping set-up is shown in Fig. 8. Examples of quasi-flattop pulses thus produced as measured by a sampling oscilloscope are shown in Fig. 9. From the trace sharpness of the falling edge, the jitter between the twin outputs is estimated at less than 20 ps. The voltage has been. scaled to correspond to that launched onto the MCP in the absence of any impedance mismatch losses. The dashed curve with increased risetime corresponds to the case of including a dc blocking capacitor between MCP and pulser so as to avoid damaging the pulser when biasing the MCP. A few $100 \mathrm{~V}$ of reverse dc bias is predicted to be effective and necessary for reducing the effective rise-times and fall-times of the gain. 


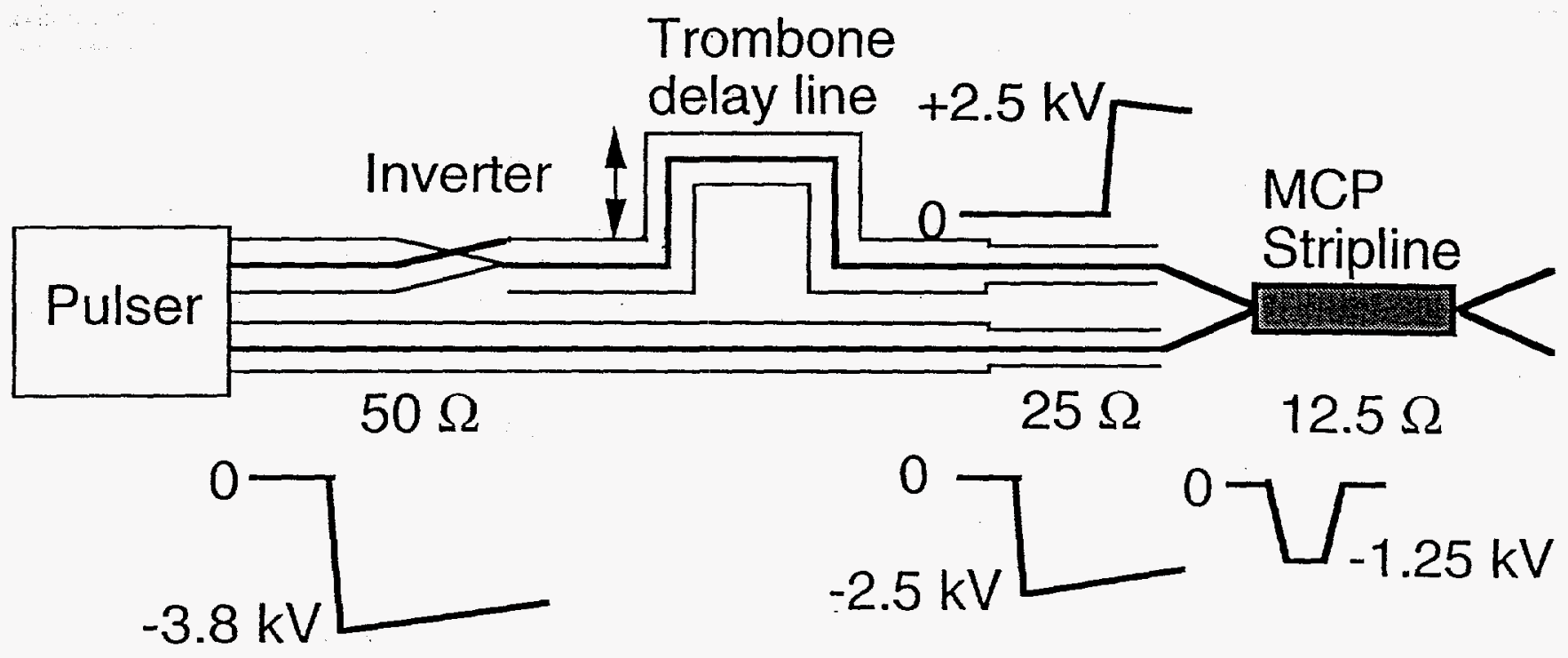

Figure 8 Experimental set-up for creating flattop gating pulses.

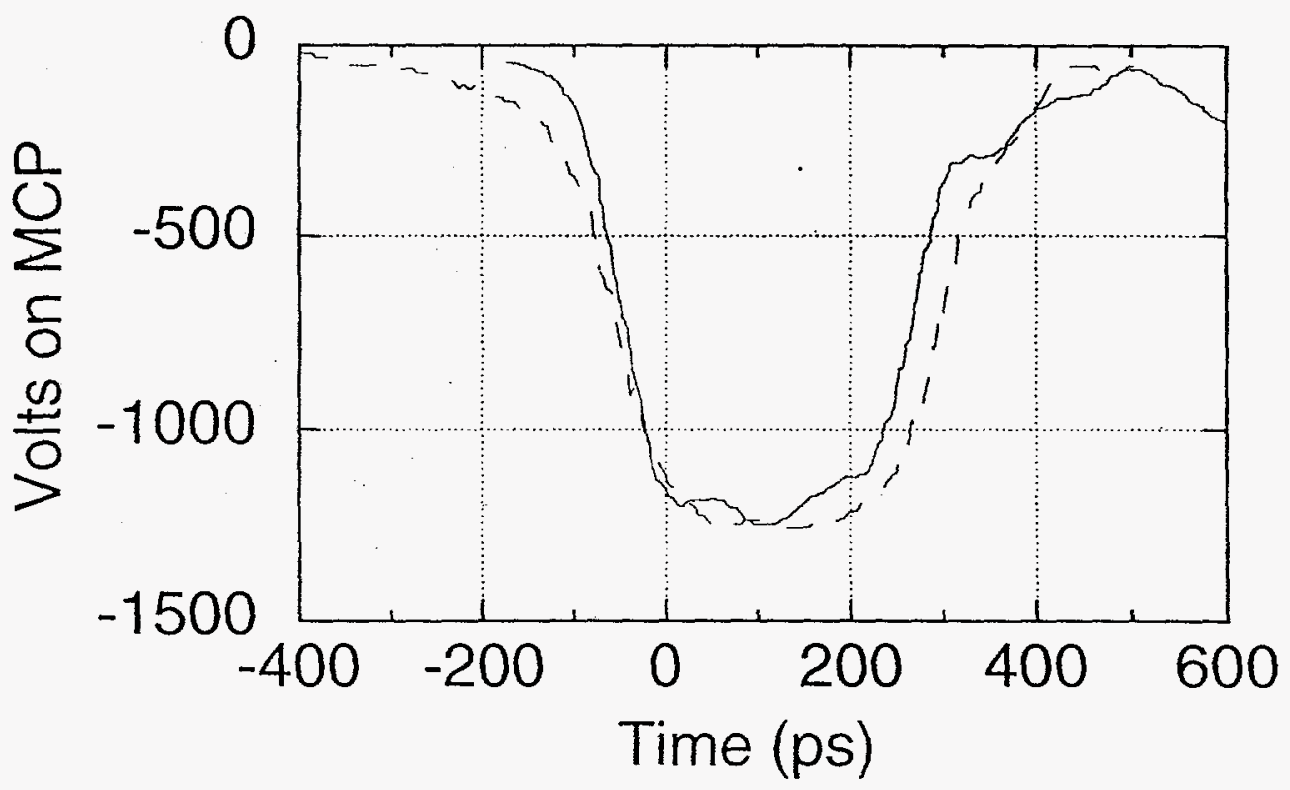

Figure 9 Examples of sampling oscilloscope measurements of flattop profiles with (dashed curve) and without (solid curve) $25 \Omega \mathrm{dc}$ blocking capacitor. Maximum voltage attainable was $-1250 \mathrm{~V}$.

\subsection{Gate profile modelling}

Results of the gain profile modelling without dispersion are shown in Fig. 10. The standard $\mathrm{LD}=45,10 \mu \mathrm{m}$ pore MCP characteristics given in section 2.3 were used in the calculations. The voltage profiles are analytic approximations to those shown in Fig. 9, and are constructed by computationally recombining opposite polarity, 90 ps HWHM Gaussian rise-time step pulses. $+300 \mathrm{~V}$ of reverse dc bias has been added to the pulsed voltage in the modelling to sharpen the voltage rise- and fall- 
times. A sequence of paired voltage and gain profiles are plotted for 20 ps increments in the variable delay between the recombined step pulses. The peak gain and FWHM drop rapidly with decreasing voltage FWHM. Between the maximum and minimum delays ( 400 and $320 \mathrm{ps,} \mathrm{respectively),} \mathrm{the} \mathrm{FWHM} \mathrm{has} \mathrm{decreased} \mathrm{from} 80$ ps to 15 ps while the peak gain has dropped by $90 \%$. However, at a 15 ps gate duration setting, the gain drops to zero or the gate duration doubles for as small as $10 \%$ variations in peak voltage amplitude or \pm 20 ps variations in voltage FWHM.

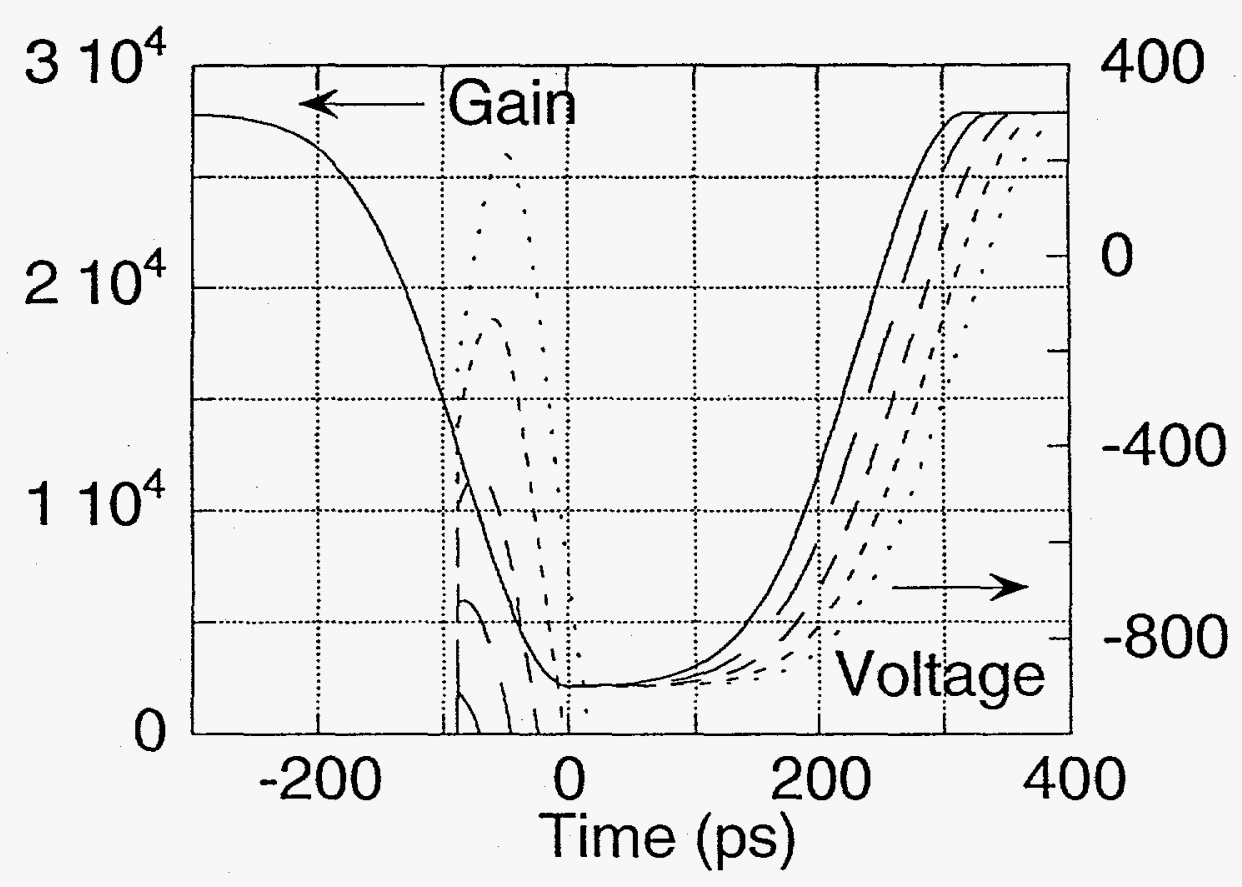

Figure 10 Simulation results for gate profiles using a time dependent discrete dynode model. The assumed voltage profiles which closely approximate measured profiles are also shown. The different voltage curves and associated gain profiles are for 20 ps increments in voltage FWHM. Peak pulsed voltage is $-1250 \mathrm{~V}$, reverse DC bias is $+300 \mathrm{~V}$ and minimum voltage FWHM corresponds to a trombone delay of $320 \mathrm{ps.}$

\subsection{Measured gain profiles}

The minimum gate FWHM measured to date for an $L / D=45,10 \mu \mathrm{m}$ pore $\mathrm{MCP}$, pulsed by flat-top gate profiles, is 50ps FWHM, consistent with estimates of transit time broadening. The peak gain at that setting is $\approx 1000 \mathrm{x}$ weaker than the case shown on Fig. 5 for example, at the practical limit for ICF experiments. However, an LD $=40 \mathrm{MCP}$ with half the thickness and hence half the predicted transit time dispersion is now routinely available ${ }^{12}$ for more flattop gate testing.

\section{SUMMARY}

High contrast (down to 1 part in $10^{4}$ ) measurements of gain profiles for MCP-based $x$-ray framing cameras used in implosion imaging have been performed. The results show little distortion due to reflections from the ends of the microstrips, but some degradation in profile contrast attributed to voltage dispersion during gate propagation across the microstrip. The profiles are well modelled by a simple time-dependent discrete dynode model down to the $1 \%$ signal level, consistent with negligible broadening due to transit time dispersion for $100 \mathrm{ps} \mathrm{FWHM} \mathrm{gate} \mathrm{durations.} \mathrm{Reproducible,}>1 \mathrm{kV}$ flat-top voltage profiles of continuously variable duration were produced to test the ultimate limitations on the shortest gain profiles achievable with standard 450- $\mu$ m-thick MCPs. A minimum gate time of $50 \mathrm{ps}$ was achieved, comparable to estimates of transit time dispersion and at variance with sub-20ps gate time predictions ignoring transit time dispersion. 


\section{ACKNOWLEDGMENTS}

We thank J.D. Kilkenny and J.D. Wiedwald for useful discussions. This work was performed under the auspices of the U.S. Department of Energy by Lawrence Livermore National Laboratory under contract No. W-7405-ENG-48.

\section{REFERENCES}

1. M. Katayama, M. Nakai, T. Yamanaka, Y. Izawa and S. Nakai, "Multiframe x-ray imaging system for temporally and spatially resolved measurements of imploding inertial confinement fusion targets," Rev. Sci. Instrum. 62 (1991) 124.

2. D.K. Bradley, P.M. Bell, J.D. Kilkenny, R. Hanks, O. Landen, P.A. Jaanimagi, P.W. McKenty, and C.P. Verdon, "High-speed gated x-ray imaging for ICF target experiments," Rev. Sci. Instrum. 63 (1992) 4813.

3. P.M. Bell, J.D. Kilkenny, G. Power, R. Bonner, and D.K. Bradley, "Multiframe x-ray images from a single meander stripline coated on a microchannel plate," in Ultrahigh-and High Speed Photography, Photonics, and Videography "89, SPIE Vol. 1155 (SPIE, Bellingham WA, 1989), p. 430.

4. F. Ze, R.L. Kauffman, J.D. Kilkenny, J. Wiedwald, P.M. Bell, R. Hanks, J. Stewart, D. Dean. J. Bower, and R. Wallace, "A new multichannel soft x-ray framing camera for fusion experiments," Rev. Sci. Instrum. 63 (1992) 5124.

5. J.D. Kilkenny, "High speed proximity focused x-ray cameras," Lasers and Part. Beams 9 (1991) 49.

6. E.H. Eberhardt, "Gain model for microchannel plates," Appl. Opt. 18 (1979) 1418.

7. O.L. Landen, P.M. Bell, J.A. Oertel, J.J. Satariano, and D.K. Bradley, "Gain uniformity, linearity, saturation and depletion in gated microchannel-plate $x$-ray framing cameras" in Ultrahigh-and High-Speed Photography, Videography, and Photonics '93, SPIE Vol. 2002 (SPIE, Bellingham WA, 1993), p. 2.

8. P.M. Bell, B.A. Hammel, J.D. Kilkenny, R.E. Turner, and C.L. Lewis, "Modelling and measurements of sub 100 ps gating with microchannel plates" in Ultrahigh-and High Speed Photography, Photonics, and Videography '89, SPIE Vol. 1155 (SPIE, Bellingham WA, 1989), p. 415.

9. P.M. Bell, J.D. Kilkenny, R.Hanks, and O.L. Landen, "Measurements with a 35 ps gate time microchannelplate camera" in Ultrahigh- and High Speed Photography, Photonics, and Videography '90, SPIE Vol. 1346 (SPIE, Bellingham WA, 1990), p. 456.

10. M.D. Perry, O.L. Landen, R. Ettlebrick, and J. Weston, "Design and performance of a high-power, synchronized Nd:YAG-dye laser system," Opt. Lett. 14 (1989) 42.

11. O.L. Landen, R.A. Lerche, R.G. Hay, B.A. Hammel, D. Kalantar, and M.D. Cable, "An x-ray technique for precision laser beam synchronization," to appear in Rev. Sci. Instrum.

12. P.M. Bell, J.D. Kilkenny, O.L. Landen, D.K. Bradley, R.G. Watt, and J. Oertel, "Implementation of 40-ps high-speed gated-microchannelplate-based $\mathrm{x}$-ray framing cameras on reentrant SIMs for Nova," this proceedings. 